\title{
AVALIAÇÃO DO DESEMPENHO DA CLASSIFICAÇÃO DO USO E COBERTURA DA TERRA A PARTIR DE IMAGENS LANDSAT 8 E RAPIDEYE NA REGIÃO CENTRAL DO RIO GRANDE DO SUL.
}

\author{
PERFORMANCE EVALUATION OF LAND USE AND COVER CLASSIFICATION USING \\ LANDSAT 8 AND RAPIDEYE IMAGES IN THE CENTRAL REGION OF RIO GRANDE DO \\ SUL.
}

\author{
Túlio Barroso QUEIROZ¹, Rita dos Santos SOUSA², Talita BALDIN², Fábio de Jesus \\ BATISTA $^{2}$, Juliana MARCHESAN ${ }^{2}$, Letícia Daiane PEDRALI ${ }^{2}$, Rudiney Soares PEREIRA ${ }^{2}$ \\ (1) Programa de Pós-graduação em Ciência Florestal da Universidade Estadual Paulista - UNESP - Botucatu, SP. \\ Email:tulio-bq@hotmail.com \\ (2) Programa de Pós-graduação em Engenharia Florestal da Universidade Federal de Santa Maria (UFSM), Santa Maria - RS. Emails: \\ ritasousa.ufsm@gmail.com; baldintalita@gmail.com; fabiodejesusbatista@gmail.com; marchesan.ju@gmail.com; \\ lelepedrali@gmail.com; rudiney.s.pereira@gmail.com
}

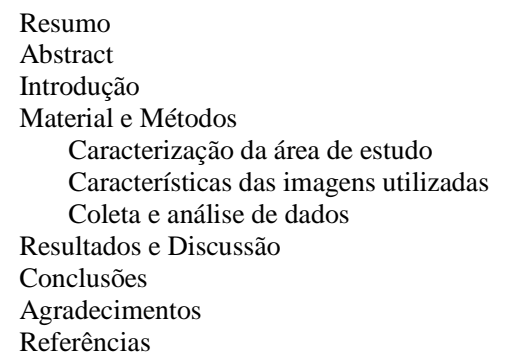

RESUMO - Imagens digitais com diferentes resoluções espaciais podem ser utilizadas para conhecer a dinâmica do uso e cobertura da superfície terrestre. O Planalto Meridional Sul-rio-grandense apresenta diversificada ocupação do solo, assim, imagens obtidas dessa região podem apresentar comportamento espectral diferenciado. Desse modo, este estudo teve como objetivo avaliar a classificação temática do uso e cobertura da terra na região central do Rio Grande do Sul por meio de imagens de média (OLI/Landsat 8) e alta (REIS/RapidEye) resolução espacial. As classes temáticas (CT) foram identificadas e quantificadas com auxílio do software Spring v.5.1.8 e avaliadas pela distribuição de 200 pontos por meio de amostragem aleatória simples com o uso do QGis v.2.10.1. Na área estudada as CT's mais representativas em ambas as imagens foram agricultura e floresta nativa. As classes menos representativas para o Landsat 8 foram água e solo exposto e para o RapidEye água e floresta plantada. $\mathrm{O}$ sistema de classificação resultou em quociente Kappa de 0,59\% (bom) e 0,73\% (muito bom), para as imagens Landsat 8 e RapidEye, respectivamente. A utilização de ambas as imagens foi satisfatória para a classificação do uso e cobertura da terra adotada neste estudo, entretanto, as imagens RapidEye são mais adequadas para classificações que necessitam maior detalhamento da área.

Palavras-chave: cobertura florestal, geoprocessamento, sensoriamento remoto.

\begin{abstract}
Digital images with different spatial resolutions can be used to understand the dynamics of use and coverage of the Earth's surface. The Southern Plateau Sul-rio-grandense presents diversified land use, thus, images obtained from that region may contain distinct spectral behavior. Therefore, this study aimed to evaluate the thematic classification of land use and cover in the central region of Rio Grande do Sul through images of medium (Landsat 8/OLI) and high (REIS/RapidEye) spatial resolution. The thematic classes (CT) were identified and quantified with software Spring v. 5.1.8 and evaluated taking into account the distribution of 200 random points using QGis v. 2.10.1. In the studied area the CT's most representative in both sensors were agriculture and native forest. The classes less representative for the Landsat 8 were water and exposed soil and for the RapidEye were water and planted forest. The classification system resulted in Kappa coefficient of 0,59\% (good) and 0,73\% (very good), to Landsat 8 and RapidEye images, respectively. The use of both images has been satisfactory for the land use and land cover classification employed in this study, however, RapidEye images are more suitable for classifications that require greater detail of the area.
\end{abstract}

Keywords: forest cover, geoprocessing, remote sensing.

\section{INTRODUÇÃO}

As técnicas de sensoriamento remoto e geoprocessamento tornaram-se ferramentas úteis e indispensáveis para o monitoramento da dinâmica de uso e ocupação do solo, uma vez que proporcionam maior agilidade no processamento e atualização de dados com menor custo (Vaeza et al., 2010).

A classificação de imagens multiespectrais de sensores orbitais consiste em uma metodologia usada para caracterização, comparação de paisagens, gestão ambiental e ordenamento do território com aplicações na modelagem e monitoramento das mudanças na paisagem (Stevic et al., 2016).

A classificação de imagens pode ser definida como o processo que permite transformar uma imagem numérica multiespectral, constituída por diferentes bandas, em uma carta temática, no decorrer do qual os objetos sobre a superfície terrestre são agrupados e 
identificados de acordo com as suas características espectrais, espaciais ou temporais, atribuindo cada pixel a uma determinada classe ou categoria previamente definida pelo pesquisador (Lillesand \& Kiefer, 1994; Meneses \& Sano, 2012).

Os métodos de classificação são divididos em dois grupos: os supervisionados e os não supervisionados.

A classificação supervisionada tem maior precisão, sendo obtida a partir dos dados de campo com base no conhecimento prévio das áreas e dos tipos de ocupação do solo que se pretendem levantar e classificar (Oliveira et al., 2014).

Desta forma a imagem é classificada nas classes de interesse pré-definidas, onde o algoritmo necessita ser treinado para poder distinguir as classes uma das outras (Meneses \& Sano, 2012).

$\mathrm{Na}$ classificação não supervisionada os pixels são agrupados em classes, de acordo com as suas características, determinadas a partir de cálculos estatísticos (Moraes, 1999). Normalmente é utilizada quando não se têm informações sobre o número e natureza das classes de alvos presentes na área, ou quando se pretende fazer uma classificação exploratória (Meneses \& Sano, 2012).

A validação dos dados gerados por meio de processamento digital de imagens de satélite é de fundamental importância, evitando interpretações errôneas.

A acurácia dos dados geográficos deve ser observada considerando três pontos fundamentais: a posição dos dados; a época de aquisição dos dados; e a temática investigada (Lobão et al., 2005).

Nesta lógica, três tipos de erros diferentes devem ser avaliados: o erro posicional, o erro temporal e o erro temático (Antunes et al., 2004). Para avaliação de erros temáticos podem ser utilizados índices como, por exemplo, o Kappa ou o Pabak (Prevalence and Bias Adjusted Kappa).

As imagens derivadas de produtos de sensoriamento remoto são excelentes fontes para produzir mapas de uso e cobertura da terra (Leão et al., 2007).

No entanto, segundo Garofalo et al., (2015) ainda existem lacunas de estudos que visem avaliar comparativamente a acurácia de classificadores digitais aplicados às imagens Landsat $8 \mathrm{em}$ ambientes tropicais.

Desse modo, o objetivo deste trabalho foi avaliar o desempenho da classificação temática do uso e cobertura da terra na região central do Rio Grande do Sul por meio de imagens de resolução espacial média (OLI/Landsat 8) e alta (REIS/RapidEye).

\section{MATERIAIS E MÉTODOS UTILIZADOS}

\section{Caracterização da área de estudo}

A área de estudo é composta por um bloco geográfico localizado na região central do Estado do Rio Grande do Sul, totalizando uma área de aproximadamente $1.175,5 \mathrm{Km}^{2}$.

O bloco está posicionado entre as coordenadas $29^{\circ} 19^{\prime} 48.0^{\prime \prime}$ e $29^{\circ} 41^{\prime} 0.1^{\prime \prime}$ ' de latitude sul e $53^{\circ} 18^{\prime} 46.0^{\prime \prime}$ e $53^{\circ} 41^{\prime} 33.0^{\prime \prime}$ 'de longitude oeste, abrangendo os municípios de Agudo, Dona Francisca, Faxinal do Soturno, Ivorá, Júlio de Castilhos, Nova Palma, Pinhal Grande, Restinga Seca, Santa Maria, São João do Polêsine e Silveira Martins (Figura 1).

Constituído pelas regiões fisiográficas do Planalto Meridional Sul-rio-grandense, com relevo tabular muito escavado pelos rios, e Depressão Central (Streck et al., 2008).

A região abrange os biomas Mata Atlântica e Pampa. De acordo com a classificação de Köppen o clima é Subtropical Úmido (Cfa), com temperaturas médias que variam entre $16^{\circ} \mathrm{C}$ e $20^{\circ} \mathrm{C}$ e precipitação média que varia de $1.600 \mathrm{~mm}$ a $1900 \mathrm{~mm}$ distribuídas ao longo do ano (Alvares et al., 2013).

A base da economia nesta região é a agropecuária, configurando assim a presença de grandes e médias propriedades ocupadas por lavouras de arroz e soja, pequenas propriedades de origem colonial com produção diversificada e áreas de campos voltadas à pecuária (FEE, 2014). Segundo Kersten et al., (2014), a região apresenta características propícias para o cultivo do arroz irrigado, por contar com margens planas ao longo do rio e áreas sujeitas a inundação, ideais para este cultivo.

\section{Características das imagens utilizadas}

Foram utilizadas imagens de dois satélites distintos, Landsat 8 e RapidEye. As características técnicas das imagens trabalhadas são observadas abaixo (Tabela 1). 


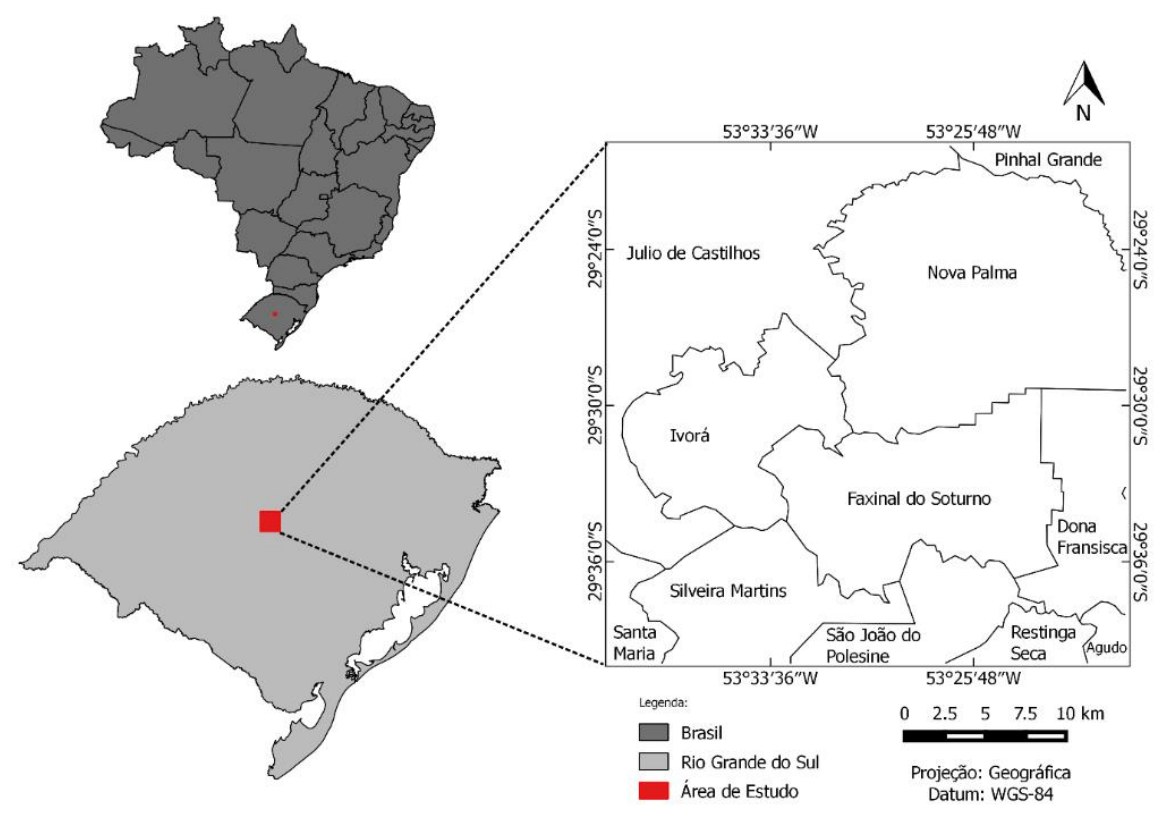

Figura 1 - Localização da área de estudo, Estado do Rio Grande do Sul, Brasil.

Tabela 1 - Características técnicas dos satélites Landsat 8 e RapidEye.

\begin{tabular}{|c|c|c|}
\hline Órbita & Heliossíncrona com $705 \mathrm{~km}$ de altitude & Heliossíncrona com $630 \mathrm{~km}$ de altitude \\
\hline Passagem Equador & $+/-10 h$ & $+/-11 \mathrm{~h}$ \\
\hline Tipos de sensores & $\begin{array}{l}\text { Operacional land imager }(O L I) \text { : bandas } \\
\text { multiespectrais } 1-7 \text { e } 9 \\
\text { Operacional land imager }(\text { OLI): banda } \\
\text { pancromática } 8 \\
\text { Thermal infrared sensor }(\text { TIRS): bandas } \\
10 \text { e } 11\end{array}$ & Multiespectral (pushbroom imager) \\
\hline \multirow{11}{*}{ Bandas } & 1. Coastal/Aerosol $(0,43-0,45 \mu \mathrm{m})$ & 1. Azul $(0,44-0,51 \mu \mathrm{m})$ \\
\hline & 2. Azul $(0,45-0,51 \mu \mathrm{m})$ & 2. Verde $(0,52-0,59 \mu \mathrm{m})$ \\
\hline & 3. Verde $(0,53-0,59 \mu \mathrm{m})$ & 3. Vermelho $(0,63-0,68 \mu \mathrm{m})$ \\
\hline & 4. Vermelho $(0,64-0,67 \mu \mathrm{m})$ & 4. Red-Egde $(0,69-0,73 \mu \mathrm{m})$ \\
\hline & 5. NIR $(0,85-0,88 \mu \mathrm{m})$ & 5. Infr. próximo $(0,76-0,85 \mu \mathrm{m})$ \\
\hline & 6. SWIR-1 (1,57-1,65 $\mu \mathrm{m})$ & - \\
\hline & 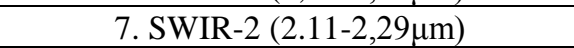 & - \\
\hline & 8. PAN $(0,50-0,68 \mu \mathrm{m})$ & - \\
\hline & 9. Cirrus $(1,36-1,38 \mu \mathrm{m})$ & - \\
\hline & 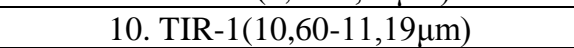 & - \\
\hline & 11. TIR-2 $(11,50-12,51 \mu \mathrm{m})$ & - \\
\hline Tamanho do Pixel & $\begin{array}{c}\text { 100m (TIR 1 e 2) } \\
\text { 30m (demais bandas) } \\
15 \mathrm{~m} \text { (PAN) }\end{array}$ & $\begin{array}{c}6,5 \mathrm{~m} \text { (nadir) } \\
5 \mathrm{~m}(\text { ortorretificado })\end{array}$ \\
\hline Tamanho da imagem & $185 \mathrm{~km} \times 185 \mathrm{~km}$ & $77 \mathrm{~km}$ de largura \\
\hline Tempo de revisita & 16 dias & 5,5 dias (no nadir) \\
\hline DATUM horizontal & WGS84 & WGS84 \\
\hline Resolução radiométrica & 16 bits & 12 bits \\
\hline Fonte de pesquisa & USGS (2016) & Felix et al. (2009) \\
\hline
\end{tabular}

\section{Coleta e análise de dados}

Foi utilizada uma imagem digital de média resolução espacial $(30 \mathrm{~m})$ do sensor OLI (Operational Land Imager), a bordo do satélite Landsat 8, constituída pelas bandas 2, 3, 4, 5, 6 e 7, de outubro de 2013, disponibilizada gratuitamente pelo USGS (United States Geological Survey) no site http://earthexplorer.usgs.gov/.
A imagem de alta resolução espacial $(5 \mathrm{~m})$ foi obtida pelo sensor REIS (RapidEye Earth Imaging System), satélite RapidEye, bandas espectrais 1, 2, 3, 4 e 5, de setembro de 2013, cedidas pelo projeto "Tecnologia de monitoramento automatizado da cobertura florestal em áreas de floresta estacional decidual na região Centro-Serra do RS" desenvolvido pelo Laboratório de 
Sensoriamento Remoto (LABSERE) da Universidade Federal de Santa Maria em parceria com o Sindicato Interestadual da Indústria do Tabaco.

Os programas computacionais utilizados foram o SPRING versão 5.1.8 (Câmara et al., 1996), para processamento e análise das imagens, o QGis versão 2.10.1 para geração dos pontos aleatórios e elaboração dos mapas finais e o Google Earth Pro para a verificação da veracidade da classificação das imagens.

Primeiramente foi construído um banco de dados no SPRING, por meio da importação das imagens e dos arquivos vetoriais referentes aos limites de classe temática, sendo utilizada a projeção LATLONG e Datum WGS84 (World Geodetic System).

Foi elaborada a composição falsa-cor RGB (Red, Green e Blue) com as bandas espectrais 6, 5 e 4 para as imagens do satélite Landsat e 5, 4 e 3 para o RapidEye, onde se obteve a melhor discriminação dos alvos.

Com base no Manual do Uso da Terra do IBGE (2013), foram definidas seis classes temáticas (CT's) de uso e cobertura da terra: "Floresta Nativa", "Floresta Plantada", "Campo", "Agricultura", "Solo Exposto" e "Água”.

Em seguida foi aplicada a técnica de segmentação por "regiões de crescimento", com similaridade 100 e área 10, tanto para as imagens do satélite Landsat 8 quanto para o RapidEye, pois foram os parâmetros que melhor se adaptaram para a área de estudo. A classificação foi de forma supervisionada utilizando o algoritmo Bhattacharya, com grau de aceitação $99 \%$.

A medida da distância de Bhattacharya foi empregada na separabilidade estatística entre pares de classes espectrais, ou seja, distância média entre as distribuições de probabilidades de classes espectrais (INPE, 2015).

As áreas foram quantificadas com auxílio da função cálculo de áreas do programa SPRING, sendo este efetuado a partir da contagem do número de pixels classificados em cada uma das classes de uso e cobertura da terra, considerando a respectiva resolução espacial de cada imagem (Bolfe et al., 2004).

Foram utilizadas 200 amostras, distribuídas por meio de amostragem aleatória simples, na área de estudo para avaliação da classificação supervisionada das imagens.

No intuito de identificar a fidedignidade das categorias de uso e cobertura da terra, estes pontos foram utilizados na construção da matriz de erros das imagens classificadas, segundo metodologia proposta por Congalton (1991).

Geralmente, a verificação é realizada em campo, no entanto, devido à inviabilidade de acesso à área e defasagem temporal da tomada das imagens foi utilizada como documento de referência, uma imagem disponibilizada pelo programa Google Earth Pro, com data de 15/08/2013 e resolução desconhecida.

A validação da classificação gerada foi realizada pelo índice Kappa (Congalton \& Green, 1999). Este índice, segundo Brites (1996), considera que tanto os produtos gerados como o documento de referência possuem o mesmo grau de verdade e ainda todos os elementos da matriz de erros, estimando assim a soma das colunas e linhas marginais (Cohen, 1960), conforme expressão a seguir:

$$
\mathrm{K}=\frac{n \sum_{i=1}^{k} n_{i i}-\sum_{i=1}^{k} n_{i+} n_{+i}}{n^{2}-\sum_{i=1}^{k} n_{i+} n_{+i}}
$$

Onde: $\mathrm{k}=$ número de linhas da matriz de erros; $\mathrm{n}=$ número total de observações (amostras); $\mathrm{n}_{\mathrm{ii}}=$ número de observações na linha $\mathrm{i}$ e coluna $\mathrm{i} ; \mathrm{n}_{\mathrm{i}+}=$ total da linha $\mathrm{i} ; \mathrm{n}_{+\mathrm{i}}=$ total da coluna $i$.

Os valores Kappa obtidos foram comparados aos conceitos de desempenho estabelecido por Congalton \& Green (1999) (Tabela 2).

A finalização dos processos de validação foi realizada a partir da matriz de confusão onde se calcula a exatidão global dos mapas (Chuviecco, 1995).

Essa matriz é uma modalidade de tabela de contingência, a partir da qual foram extraídas informações quantitativas acerca do processo de verificação, conforme proposto por Ruiz (1995). A exatidão global do mapa relaciona os elementos da diagonal principal com o total de pontos amostrados (Chuviecco, 1995).

$$
\mathrm{Fm}=\frac{\sum \mathrm{x}_{\mathrm{ii}}}{\sum \sum \mathrm{x}_{\mathrm{ij}}}
$$

Onde: $\mathrm{Fm}=$ exatidão global; $\mathrm{x}_{\mathrm{ii}}=$ elementos da diagonal; $\Sigma \Sigma \mathrm{x}_{\mathrm{ij}}=$ soma dos elementos. 
Tabela 2 - Critérios utilizados na avaliação do grau de concordância da classificação temática com base no índice Kappa. Fonte: Congalton \& Green (1999).

\begin{tabular}{c|c}
\hline Valor do Kappa & Concordância \\
\hline $\mathrm{K} \leq 0$ & Péssimo \\
\hline $0,0<\mathrm{K} \leq 0,2$ & Ruim \\
\hline $0,2<\mathrm{K} \leq 0,4$ & Razoável \\
\hline $0,4<\mathrm{K} \leq 0,6$ & Bom \\
\hline $0,6<\mathrm{K} \leq 0,8$ & Muito Bom \\
\hline $0,8<\mathrm{K}<1,0$ & Excelente \\
\hline
\end{tabular}

A precisão da categoria individual foi obtida por meio da razão do número total de amostras classificadas corretamente naquela categoria pelo número total de amostras (Figueiredo \& Vieira, 2007; Ruiz, 1995).

Assim foi necessário considerar as células marginais da matriz para dimensionar os erros. As células marginais nas linhas indicam o número de pixels que não foram incluídos em determinada categoria, isto é, expressam o erro conhecido por omissão. Por outro lado, as células nas diagonais das colunas representam os pixels que também não foram incluídos em nenhuma categoria, expressando o erro de comissão (Chuviecco, 1995).

A omissão se refere a uma definição imperfeita da categoria e a comissão a uma delimitação excessiva da categoria (Ferreira, et. al. 2007).

$\mathrm{O}$ erro de omissão $\left(\mathrm{EO}_{\mathrm{i}}\right)$ e o erro de comissão $\left(\mathrm{EC}_{\mathrm{j}}\right)$ foram calculados para cada classe temática da classificação conforme expressões abaixo.

$$
\mathrm{E}_{i}=\frac{\sum \mathrm{x}_{\mathrm{ij}}-x_{i i}}{\sum \mathrm{x}_{\mathrm{ij}}} \quad \mathrm{E} C_{j}=\frac{\sum \mathrm{x}_{\mathrm{ij}}-x_{j j}}{\sum \mathrm{x}_{\mathrm{ij}}}
$$

Onde:

$\Sigma \mathrm{x}_{\mathrm{ij}}-\mathrm{X}_{\mathrm{ii}}=$ soma dos resíduos por linha;

$\Sigma \mathrm{x}_{\mathrm{ij}}-\mathrm{X}_{\mathrm{jj}}=$ soma dos resíduos por coluna;

$\Sigma \mathrm{x}_{\mathrm{ij}}=$ marginal da linha ou coluna.

Os erros de omissão e comissão em porcentagens foram denominados, respectivamente, de risco do produtor e de risco do usuário.

\section{RESULTADOS E DISCUSSÃO}

No processamento digital das imagens foram observadas diferenças no comportamento espectral para o uso e cobertura da terra. Por outro lado, a distribuição aleatória da amostragem acarretou na má distribuição das amostras ao longo das CT's nas duas imagens, não sendo distribuídas homogeneamente, (Landsat 8: $\mathrm{FN}=81$ amostras; $\mathrm{FP}=04 ; \mathrm{AG}=68$; $\mathrm{SE}=13 ; \mathrm{CA}=31 ; \mathrm{A} g=03$; e RapidEye: $\mathrm{FN}=74$ amostras; $\mathrm{FP}=01 ; \mathrm{AG}=44 ; \mathrm{SE}=27 ; \mathrm{CA}=49$; $\hat{A} g=05)$. Congalton (1991) sugeriu o tamanho mínimo de 50 amostras para cada categoria em áreas que tenham uma abrangência territorial de até aproximadamente $4.050 \mathrm{Km}^{2}$.

Os padrões de distribuição espacial nos dois formatos de imagens foram semelhantes, entretanto as imagens RapidEye evidenciaram maior heterogeneidade (Figura 2). Resultados semelhantes foram observados por Naesset et al. (2016), estudando uma floresta na Tasmânia com imagens ALS, SAR interferométrico (InSAR), RapidEye, Landsat e ALOS PALSAR banda L (radar). Os autores observaram que as imagens RapidEye apresentaram as mesmas tendências, isto é, um maior grau de detalhamento devido ao GSD (Ground Sample Distance) das imagens ser menor que as demais.

A classificação realizada nas imagens obtidas a partir do Landsat 8 demonstrou que o componente de maior ocupação do uso e cobertura da terra correspondeu a agricultura 53.111,34ha (45,18\%). Entretanto, nas imagens RapidEye a maior área classificada foi de floresta nativa, correspondendo a 43.886,12ha $(37,33 \%)$. As classes de água e floresta plantada foram as de menor representatividade em ambos os satélites (Tabela 3).

Foi registrada menor área agrícola e maior área de floresta nativa na imagem RapidEye em relação a Landsat 8. Segundo Naesset et al., (2016) o modelo RapidEye consegue captar a heterogeneidade espacial dentro de parcelas em termos de árvores dispersas e grupos de árvores. $\mathrm{O}$ mesmo raciocínio pode ser feito ao se considerar a agricultura e campo, 
justificando as diferenças existentes nestas duas classes. Além disso, as imagens apresentam diferentes respostas devido às suas distintas especificações. Em estudo realizado com imagens do sensor OLI e MODIS, Zhou et al.,
(2016), reportaram diferenças nas classificações das mesmas áreas de cultivo de arroz $\left(1.373 \mathrm{~km}^{2}\right.$ e $1.076 \mathrm{~km}^{2}$, respectivamente), devido a classificação errônea de zonas húmidas naturais como áreas arrozeiras.

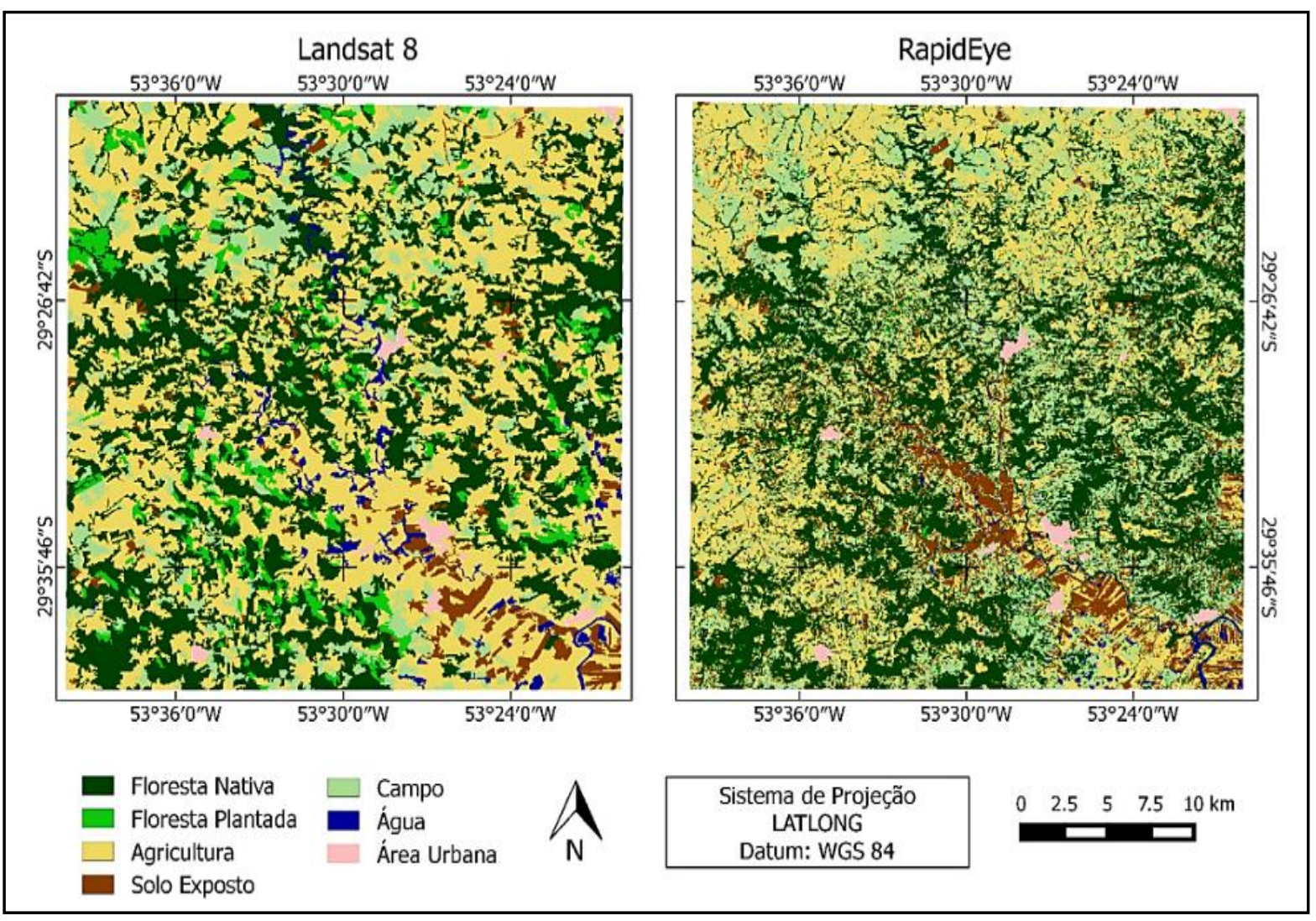

Figura 2 - Mapa temático de uso e cobertura da terra com base nas imagens Landsat 8 (out/2013) e RapidEye (set/2013) da região Central do Rio Grande do Sul.

Tabela 3 - Quantificação das classes de uso e cobertura da terra da região Central do Rio Grande do Sul, com base nas imagens Landsat 8 (out/2013) e RapidEye (set/2013).

\begin{tabular}{l|r|r|r|r}
\hline \multirow{2}{*}{ Uso da terra } & \multicolumn{2}{c|}{ Landsat 8 } & \multicolumn{2}{c}{ RapidEye } \\
\cline { 2 - 5 } & \multicolumn{1}{c|}{ ha } & \multicolumn{1}{c}{$\%$} & \multicolumn{1}{c}{ ha } & \multicolumn{1}{c}{$\%$} \\
\hline Agricultura & $53.111,34$ & 45,18 & $34.082,30$ & 28,99 \\
\hline Floresta Nativa & $41.699,72$ & 35,47 & $43.886,12$ & 37,33 \\
\hline Campo & $10.623,51$ & 9,04 & $27.725,72$ & 23,59 \\
\hline Floresta Plantada & $5.822,91$ & 4,95 & 442,25 & 0,38 \\
\hline Solo Exposto & $4.018,32$ & 3,42 & $10.162,18$ & 8,65 \\
\hline Água & $2.271,96$ & 1,93 & $1.249,18$ & 1,06 \\
\hline Total & $\mathbf{1 1 7 . 5 4 7 , 7 6}$ & $\mathbf{1 0 0}$ & $\mathbf{1 1 7 . 5 4 7 , 7 6}$ & $\mathbf{1 0 0}$ \\
\hline
\end{tabular}

Os resultados estão diretamente associados à realidade socioeconômica predominante na área de estudo. Situada na região central do Estado do Rio Grande do Sul, constitui uma zona de transição agroecológica que se estende desde o Planalto, abrangendo o rebordo do Planalto e terminando na planície aluvial. A região do rebordo, originalmente coberta por florestas, foi ocupada por imigrantes, a partir da década de 1880 .
Assim, atualmente, a região é formada por pequenas propriedades baseada na produção agropecuária, com predomínio de plantios de fumo e arroz nas várzeas e de soja no Planalto (Diesel et al., 2005).

Neumann (2004) destaca que a região é caracterizada por apresentar cultivos diversificados, destacando-se as culturas de feijão, fumo, milho e soja, além do gado de leite e de corte. Desse modo, justifica-se a 
grande porcentagem de áreas agrícolas encontradas nas classificações temáticas do presente trabalho.

Os resultados indicaram concordância entre a classificação do mapa e as imagens disponibilizadas no programa Google Earth Pro, considerada como dados de referência.
O coeficiente Kappa para a imagem Landsat 8 apresentou valor inferior $(0,59 \%)$ quando comparado com a imagem RapidEye $(0,73 \%)$. Os coeficientes determinados apontam a qualidade da classificação para Landsat 8 e RapidEye como "boa" e "muito boa", respectivamente (Tabela 4 ).

Tabela 4 - Matriz de erros de classificação temática da região Central do Rio Grande do Sul com o uso de imagens Landsat 8 (out/2013) e RapidEye (set/2013).

\begin{tabular}{|c|c|c|c|c|c|c|c|c|c|c|}
\hline \multicolumn{11}{|c|}{ Imagem Landsat 8} \\
\hline & $\mathbf{F N}$ & $\mathbf{F P}$ & $\mathbf{A G}$ & SE & CA & $\mathbf{A g}$ & & 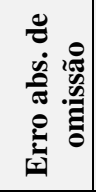 & 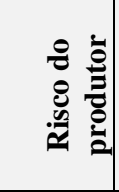 & 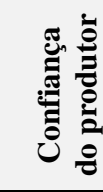 \\
\hline FN & 65 & 0 & 9 & 0 & 7 & 0 & 81 & 16 & 19,8 & 80,2 \\
\hline FP & 1 & 0 & 1 & 0 & 2 & 0 & 4 & 4 & 100,0 & 0,0 \\
\hline AG & 8 & 0 & 38 & 0 & 22 & 0 & 68 & 30 & 44,1 & 55,9 \\
\hline SE & 0 & 0 & 0 & 13 & 0 & 0 & 13 & 0 & 0,0 & 100,0 \\
\hline CA & 2 & 0 & 5 & 0 & 24 & 0 & 31 & 7 & 22,6 & 77,4 \\
\hline $\mathbf{A g}$ & 0 & 0 & 1 & 0 & 0 & 2 & 3 & 1 & 33,3 & 66,7 \\
\hline Total & 76 & 0 & 54 & 13 & 55 & 2 & 200 & - & - & - \\
\hline Erro abs. de comissão & 11 & 0 & 16 & 0 & 31 & 0 & - & - & - & - \\
\hline Risco do usuário & 14,5 & 0,0 & 29,6 & 0,0 & 56,4 & 0,0 & - & - & - & - \\
\hline Confiança do usuário & 85,5 & 100,0 & 70,4 & 100,0 & 43,6 & 100,0 & - & - & - & - \\
\hline Exatidão global & 0,71 & - & - & - & - & - & - & - & - & - \\
\hline Kappa & 0,59 & - & - & - & - & - & - & - & - & - \\
\hline \multicolumn{11}{|c|}{ Imagem Rapdieye } \\
\hline & $\mathbf{F N}$ & FP & AG & SE & CA & Ag & 苞 & 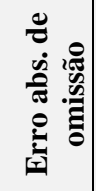 & 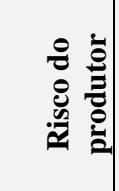 & 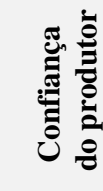 \\
\hline FN & 70 & 1 & 0 & 0 & 3 & 0 & 74 & 4 & 5,4 & 94,6 \\
\hline FP & 1 & 0 & 0 & 0 & 0 & 0 & 1 & 1 & 100,0 & 0,0 \\
\hline AG & 0 & 0 & 33 & 7 & 4 & 0 & 44 & 11 & 25,0 & 75,0 \\
\hline SE & 0 & 0 & 6 & 20 & 0 & 1 & 27 & 7 & 25,9 & 74,1 \\
\hline CA & 2 & 0 & 11 & 2 & 34 & 0 & 49 & 15 & 30,6 & 69,4 \\
\hline Ag & 0 & 0 & 1 & 1 & 0 & 3 & 5 & 2 & 40,0 & 60,0 \\
\hline Total & 73 & 1 & 51 & 30 & 41 & 4 & 200 & - & - & - \\
\hline Erro abs. de comissão & 3 & 1 & 18 & 10 & 7 & 1 & - & - & - & - \\
\hline Risco do usuário & 4,1 & 0,0 & 35,3 & 33,3 & 17,1 & 25,0 & - & - & - & - \\
\hline Confiança do usuário & 95,9 & 100,0 & 64,7 & 66,7 & 82,9 & 75,0 & - & - & - & - \\
\hline Exatidão global & 0,80 & - & - & - & - & - & - & - & - & - \\
\hline Kappa & 0,73 & - & - & - & - & - & - & - & - & - \\
\hline
\end{tabular}

A definição da matriz de erros permite observar e avaliar as divergências ocorridas entre as classes temáticas, sendo o índice Kappa um parâmetro consistente para envolver o valor final de todas as células da matriz (Bolfe et al., 2004). De forma geral, pode-se inferir que os valores foram satisfatórios, assegurando a confiabilidade do estudo.
Rudorff et al. (2007), ao realizarem uma classificação supervisionada para distinguir as áreas com e sem cultivo de soja no Estado do Rio Grande do Sul, observaram o valor estatístico kappa correspondente a 0,503, classificando-o como "bom".

Nunes \& Roing (2015) analisando o uso e ocupação do solo da bacia do Alto do Descoberto, Distrito Federal, por meio de 
classificação automática baseada em regras e lógica nebulosa, obtiveram exatidão temática muito boa, com kappa de 0,64. Nery et al. (2013), estudando a Bacia do Rio Vieira, no município de Montes Claros-MG, encontraram para as técnicas de classificação MAXVER, MAXVER - ICM e Distância Mínima Euclidiana, os índices Kappa de 0,74, 0,75 e 0,61 , respectivamente.

Congalton (1991) relatou que o uso do coeficiente Kappa é satisfatório na avaliação da precisão de uma classificação temática, pelo fato de levar em consideração toda a matriz de confusão no seu cálculo, inclusive os elementos de fora da diagonal principal, os quais representam as discordâncias na classificação (Figueiredo \& Vieira, 2007).

Os índices de exatidão global encontrados para as classificações realizadas nas imagens Landsat $8(71 \%)$ e RapidEye $(80 \%)$ foram muito bons (Tabela 4). Resultados melhores foram encontrados por Suarez \& Candeias (2012), ao classificarem uma imagem Landsat 7 onde exatidão global foi de $96 \%$ e o índice Kappa $0,94 \%$, representando um excelente grau de aceitação. Ferreira et. al. (2007), encontraram para uma imagem CBERS a exatidão de $91 \%$ e o Kappa de 0,82\%.

Segundo Bernardes (2006), o índice Kappa inclui os elementos da diagonal principal no cálculo da concordância casual fazendo com que esta concordância seja superestimada e o valor do índice reduzido.

De acordo com Brites (1996), o índice de exatidão global sempre resultará em valores mais altos que o Kappa, superestimando a avaliação da exatidão, uma vez que a mesma não considera os erros de omissão e comissão implícitos no restante da matriz.

O menor erro de omissão para a classificação da imagem RapidEye foi observado na classe Floresta Nativa (5,4\%), indicando haver inclusão errônea de poucas amostras de referência em outras categorias. Em contraponto, a classificação da imagem Landsat 8, apresentou erro de omissão de quase $20 \%$ para esta mesma classe, representando que das 81 amostras de referência 16 delas não foram incluídas nesta categoria.

Este valor foi superior ao erro de omissão encontrado por Ferreira et. al. (2007), para categoria Floresta (15\%) na classificação de uma imagem satélite Cbers2-CCD, onde os autores também utilizaram 81 amostras de referência para classe Floresta. As categorias Solo Exposto e Água não apresentaram erro de comissão.

Suarez \& Candeias (2012) mencionam valores de erros de comissão superiores aos encontrados na classificação da imagem Landsat 8 deste trabalho. Na classificação de uma imagem Landsat 7, os autores verificaram para as classes Floresta e Água os valores de $100 \%$ e $91 \%$, respectivamente.

A classe Floresta Nativa da imagem RapidEye apresentou erro de comissão de $4,1 \%$, sendo que apenas três das 70 amostras foram incluídas erroneamente. Ferreira et. al. (2007) encontraram erro de comissão de 7\% na classificação de uma imagem Cbers2 para a categoria Floresta. Neste caso, a imagem de maior resolução espacial apresentou o menor erro de comissão.

Em comparação com a Imagem Landsat 8, os erros de comissão da imagem RapidEye para as classes Agricultura, Solo exposto e Água apresentaram valores superiores $(35,3 \%, 33,3 \%$ e $25 \%$, respectivamente).

Enquanto que as classes Floresta Nativa e Campo apresentaram valores inferiores, $4,1 \%$ e $17,1 \%$ respectivamente. As diferenças na resolução espacial das imagens podem levar a uma superestimativa ou subestimativa das áreas no momento da delimitação dos polígonos das classes.

Valores semelhantes de EC\% para Floresta Plantada e Floresta Nativa são mencionados por Luz et al. (2015). Estes autores, com uso da classificação híbrida de imagens Landsat 8 e RapidEye, observaram para Floresta Nativa $\mathrm{EC}=4,08 \%$ e $\mathrm{EO}=15,60 \%$; Solo exposto $\mathrm{EC}=76,77 \%$ e $\mathrm{EO}=80,23 \%$; Agricultura $\mathrm{EC}=80,24 \%$ e $\mathrm{EO}=39,40 \%$; Corpos d'agua $\mathrm{EC}=3,72 \%$ e $\mathrm{EO}=7,82 \%$; Floresta Plantada $\mathrm{EC}=70,04 \%$ e $\mathrm{EO}=81,92 \%$.

\section{CONCLUSÕES}

O estudo aponta concordância entre a classificação do mapa e as imagens disponibilizadas no aplicativo Google Earth
Pro. A utilização de ambas as imagens foi satisfatória para o sistema de classificação de uso da terra adotado neste estudo. 
As discrepâncias espaciais e radiométricas das imagens utilizadas levaram a diferenças na classificação, sendo o mapa temático mais acurado gerado pela imagem RapidEye.

Embora não fosse objetivo do estudo avaliar a forma da distribuição dos pontos de amostra no procedimento de classificação utilizado, constatou-se que a distribuição aleatória não foi adequada na representação das classes temáticas estudadas.

\section{AGRADECIMENTOS}

À Fundação Amazônia Paraense de Amparo à Pesquisa (FAPESPA) e à Coordenação de Aperfeiçoamento de Pessoal de Nível Superior (CAPES) pela concessão de bolsa.

\section{REFERÊNCIAS}

ALVARES, C.A.; STAPE, J.L.; SENTELHAS, P.C.; GONÇALVES, J.L.M.; SPAROVEK, G. Köppen's Climate Classification Map for Brazil. Meteorologische Zeitschrift, v. 22 , n. 6, p. 711-728, 2013.

ANTUNES, A.F.B.; LIGNAU, C.; QUINTAS, M.C.L. Análise de Acurácia de mapa de uso do solo oriundo de classificação de Imagens de alta resolução. In: SIMPÓSIO DE CIÊNCIAS GEODÉSICAS E TECNOLOGIAS DA GEOINFORMAÇÃO, 1, 2004, Recife. Anais...Acessado em: 04maio2016. Disponível em: https://www.ufpe.br/cgtg/ ISIMGEO/CD/html/Fotogrametria\%20e\%20Sensoriamento\% 20Remoto/Artigos/f005.pdf.

BRITES, R.S. Verificação de exatidão em classificação de imagens digitais orbitais: efeito de diferentes estratégias de amostragem e avaliação de índices de exatidão. Viçosa, 1996, 101 p. Tese (Doutorado em Ciência Florestal) Universidade Federal de Viçosa.

BERNARDES, T. Caracterização do ambiente agrícola do Complexo Serra Negra por meio de sensoriamento remoto e sistemas de informação geográfica. Lavras, 2006, 131 p. Dissertação (Mestrado Universidade Federal de Lavras.

BOLFE, E.L.; PEREIRA, R.S.; MADRUGA, P.R. DE A.; FONSECA, E.L. Avaliação da classificação digital de povoamentos florestais em imagens de satélite através de índices de acurácia. Revista Árvore. Viçosa, v. 28, n. 1, p.85-90, 2004.

CÂMARA， G.; SOUZA， R.C.M.; FREITAS, U.M.; GARRIDO, J.; II MITSUO, F. SPRING: Integrating remote sensing and GIS by object-oriented data modelling. Computers \& Graphics, v. 20, n. 3, p. 395-403, 1996.

CHUVIECCO, E. Fundamentos de Teledetección Espacial. Madri, España: 2 ed. Ediciones Rialp, 449p. 1995.

COHEN, J. A coefficient of agreement for nominal scales. Educational and Psychological Measurement. Nova Yorque, v. 20, n.1, p. 37-46, 1960.

CONGALTON, R.G. A review of assessing the accuracy of classifications of remotely sensed data. Remote Sensing of Environment, v. 37, n.1, p. 35-46, 1991.

CONGALTON, R.G.; GREEN, K. Assessing the Accuracy of Remotely Sensed Data: Principles and Practices. Nova York, Boca Raton: Lewis Publishers, 137 p., 1999.

DIESEL, V.; LERNER, F.; SILVEIRA, P. R. C. DA.; NEUMANN, P. S.; ZARZA, G. J. G.; FREITAS, LUIS A. DOS S. DE. Caracterização da agroindústria familiar de aguardente de cana-de-açúcar na região da Quarta Colônia. In: CONGRESSO INTERNACIONAL DE DESENVOLVIMENTO RURAL E AGROINDÚSTRIA FAMILIAR, 1, 2005, São Luis Gonzaga. Anais... São Luis Gonzaga: URI, 2005, p. 315-323.

FEE - FUNDAÇÃO DE ECONOMIA E ESTATÍSTICA. Dados abertos. Disponível em: http://dados.fee.tche.br/. Acessado em: 03maio2015.

FELIX, I.M.; KASMIERCZAC, M.L.; ESPINDOLA, G.M. Rapideye: a nova geração de satélites de observação da terra. In: XIV SIMPÓSIO BRASILEIRO DE SENSORIAMENTO
REMOTO, 2009, Natal, RN, Brasil. Anais... Natal: INPE/SELPER Brasil, 2009, p. 25-30.

FERREIRA, E.; DANTAS, A.A.A.; MORAIS, A.R. Exatidão na classificação de fragmentos de matas em imagem do satélite Cbers-CCD, no munícipio de Lavras, MG. In: XIII SIMPÓSIO BRASILEIRO DE SENSORIAMENTO REMOTO, 2007, Florianópolis, SC, Brasil. Anais... Florianópolis: INPE/SELPER Brasil, 2007, p. 887-894.

FIGUEIREDO, G.C. \& VIEIRA, C.A.O. Estudo do comportamento dos índices de Exatidão Global, Kappa e Tau, comumente usados para avaliar a classificação de imagens do sensoriamento remoto. In: XIII SIMPÓSIO BRASILEIRO DE SENSORIAMENTO REMOTO, 2007, Florianópolis, SC, Brasil. Anais... Florianópolis: INPE/SELPER Brasil, 2007, p. $5755-5762$.

GAROFALO, D.F.T., MESSIAS, C.G., LIESENBERG, V., BOLFE, E.L., FERREIRA, M.C. Análise comparativa de classificadores digitais em imagens do Landsat-8 aplicados ao mapeamento temático. Pesquisa Agropecuária Brasileira, Brasília, v. 50, n. 7, p. 593-604, 2015.

IBGE - INSTITUTO BRASILEIRO DE GEOGRAFIA E ESTATÍSTICA. Manuais Técnicos em Geociências: Manual Técnico de Uso da Terra. $3^{\mathrm{a}}$ ed. Rio de Janeiro: Ministério do Planejamento, Orçamento e Gestão, no. 7, 171 p., 2013.

INPE - INSTITUTO NACIONAL DE PESQUISAS ESPACIAIS. Manuais. Disponível em: <http://www.dpi. inpe.br/spring/portugues/tutorial/ >. Acessado em: 22dez2015.

KERSTEN, D.S.; MARION, F.A.; MELLO FILHO, J.A. DE. Zoneamento ambiental por geoprocessamento das áreas propícias ao uso e ocupação do solo no município de Faxinal do Soturno - RS. Geografia Ensino e Pesquisa, v. 18, n. 1, p. 119-134, 2014.

LEÃO, C., KRUG, L.A., KAMPEL, M., FONSECA, L.M. G. Avaliação de métodos de classificação em imagens TM/Landsat e CCD/CBERS para o mapeamento do uso e cobertura da terra na região costeira do extremo sul da Bahia. In: XIII SIMPÓSIO BRASILEIRO DE SENSORIAMENTO REMOTO, 2007, Florianópolis, SC, Brasil. Anais... Florianópolis: INPE/SELPER Brasil, 2007, p. 939-946.

LILLESAND, T.M. \& KIEFER, R.W. Remote Sensing and Image Interpretation. 3rd ed. New york: John Wiley \& Sons, Inc., 750 p., 1994.

LOBÃO, J.S.B.; FRANÇA-ROCHA, W.J.S.; SILVA, A.B. Aplicação dos Índices KAPPA \& PABAK na validação da classificação automática de imagem de satélite em Feira de Santana-BA. Anais XII SIMPÓSIO BRASILEIRO DE SENSORIAMENTO REMOTO, 2005, Goiânia, GO, Brasil. Anais... Goiânia: INPE/SELPER Brasil, 2005, p. 1207-1214.

LUZ, N.B. DA; OLIVEIRA, Y.M.M. DE; ROSOT, M.A.D.; GARRASTAZÚ, M.C.; FRANCISCON, L.; JÚNIOR, H.N. DE M., FREITAS, J. V. DE. Classificação híbrida de imagens Landsat - 8 e RapidEye para o mapeamento do uso e cobertura da terra nas Unidades Amostrais de Paisagem do Inventário Florestal Nacional do Brasil. In: XVII SIMPÓSIO BRASILEIRO DE SENSORIAMENTO REMOTO, 2015, 
João Pessoa, PB, Brasil. Anais... João Pessoa: INPE/SELPER Brasil, 2015, p. 7222-7230.

MENESES, P.R. \& SANO, E.E. Classificação pixel a pixel de imagens. In: MENESES, P.R \& ALMEIDA T. DE. (Coordenadores), Introdução ao processamento de imagens de sensoriamento remoto. Brasília: UNB, CNPQ, p. 191208, 2012.

MORAES, R.M. Sensoriamento Remoto e Classificação de Imagens. Joaõ Pessoa: Departamento de Estatística, CCEN UFPB, 1999. Disponível em: <http://www.de.ufpb.br/ ronei/ procimagem/procimagem.htm >. Acessado em: 03maio2016.

NAESSET, E.; ORKA, H.O.; SOLBERG, S.; BOLLANDSAS, O.M.; HANSEN, H.E.; MAUYA, E.; ZAHABU, E.; MALIMBWI, R.; CHAMUYA, N.; OLSSON, H.; GOBAKKEN, T. Mapping and estimating forest area and aboveground biomass in miombo woodlands in Tanzania using data from airborne laser scanning, TanDEM-X, RapidEye, and global forest maps: A comparison of estimated precision. Remote Sensing of Enviroment, v. 175, p. 282300, 2016.

NERY, C.V.M., FERNANDES, F.H.S., MOREIRA, A.A., BRAGA, F.L. Avaliação das Técnicas de Classificação MAXVER, MAXVER - ICM e Distância Mínima Euclidiana de acordo com Índice Kappa. Revista Brasileira de Geografia Física, v. 06, n. 02, p. 320-328. 2013.

NEUMANN, P.S. O processo de diferenciação econômica do espaço rural e dos agricultores na região central do RS. In: FROEHLICH, J. M.; DIESEL. V. Espaço Rural e Desenvolvimento Regional: estudos a partir da região central do RS. Ijui: Ed. Da UNIJUI, p. 55-103, 2004.

NUNES, J.F. \& ROING, E.L. Análise e mapeamento do uso e ocupação do solo da bacia do alto do descoberto, DF/GO, por meio de classificação automática baseada em regras e lógica nebulosa. Revista Árvore, v.39, n.1, p. 25-36, 2015.

OLIVEIRA, G.C.; MARTINS, V.S.; FILHO, E I.F.; XAVIER, F.V. Classificação Supervisionada como Ferramenta para Avaliação da Dinâmica do Uso e Cobertura do Solo. Revista Enciclopédia Biosfera, v. 10, n. 18, p. 924-935, 2014.
RUDORFF, C.M.; RIZZI, R.; RUDORFF, B.F.T.; SUGAWARA, L.M.; VIEIRA, C.A.O. Superfícies de resposta espectro-temporal de imagens do sensor MODIS para classificação de área de soja no Estado do Rio Grande do Sul. Ciência Rural, v.37, n.1, p.118-125, 2007.

RUIZ, C.P. Elementos de teledetección. Madrid: Rama, 344 p., 1995.

STEVIĆ, D.; D.; B, I.; DOJČINOVIĆ, N.; JOKOVIĆ, J. Automated identification of land cover type using multispectral satellite images. Energy and Buildings, v. 115, p. 131-137, 2016.

STRECK, E.V.; KÄMPF, N.; DALMOLIN, R.S.D.; KLAMT, E.; NASCIMENTO, P.C.; SCHNEIDER, P.; GIASSON, E.; PINTO, L.F.S. Solos do Rio Grande do Sul. Porto Alegre: EMATER-RS/UFRGS, 222p., 2008.

SUAREZ, A.F. \& CANDEIAS, A. L. B. Avaliação de acurácia de classificação de dados de sensoriamento remoto para o município de Maragogipe. In: IV SIMPÓSIO BRASILEIRO DE CIÊNCIAS GEODÉSICAS E TECNOLOGIAS DE GEOINFORMAÇÃO, 2012, Recife, PE，Brasil: Anais... Refice: DECART/CTG/UFPE, 2012, p. 001-0010.

VAEZA, R.F.; FILHO, P.C.O.; MAIA, A.G.; DISPERATI, A.A. Uso e ocupação do solo em Bacia Hidrográfica urbana a partir de imagens orbitais de alta resolução. Floresta e Ambiente, v. 17, n. 1, p. 23-29, 2010.

UNITED STATE GEOLOGICAL SURVEY - USGS. Landsat8. Disponível em: http://landsat.usgs.gov/ landsat8.php. Acessado em: 26 maio 2016.

ZHOU, Y.; XIAO, X.; QIN, Y.; DONG, J.; ZHANG, G.; KOU, W.; JIN, C.; WANG, J.; LI, X. Mapping paddy rice planting area in rice-wetland coexistent areas through analysis of Landsat 8 OLI and MODIS images. International Journal of Applied Earth Observation and Geoinformation, v. 46, p. 1-12, 2016.

Submetido em 14 de outubro de 2016 Aceito em 7 de dezembro de 2016 